Case Report

\title{
Abdominopelvic Tuberculosis with a Frozen Section Analysis Consistent with Ovarian Cancer
}

\author{
Agrimaldo Martins-Filho, ${ }^{1}$ Paula Carolina Arvelos Crispim, ${ }^{1}$ \\ Renata Margarida Etchebehere, ${ }^{2}$ Cristina da Cunha Hueb Barata de Oliveira, ${ }^{3}$ \\ Eddie Fernando Candido Murta, ${ }^{1}$ and Rosekeila Simões Nomelini ${ }^{1}$ \\ ${ }^{1}$ Oncological Research Institute (IPON), Discipline of Gynecology and Obstetrics, Federal University of the Triângulo Mineiro, \\ Uberaba, MG, Brazil \\ ${ }^{2}$ Surgical Pathology Service, Federal University of the Triângulo Mineiro, Uberaba, MG, Brazil \\ ${ }^{3}$ Infectious Diseases Service, Federal University of the Triângulo Mineiro, Uberaba, MG, Brazil \\ Correspondence should be addressed to Rosekeila Simões Nomelini; rosekeila@terra.com.br
}

Received 12 October 2016; Accepted 9 March 2017; Published 9 April 2017

Academic Editor: Lawrence Yamuah

Copyright ( 2017 Agrimaldo Martins-Filho et al. This is an open access article distributed under the Creative Commons Attribution License, which permits unrestricted use, distribution, and reproduction in any medium, provided the original work is properly cited.

\begin{abstract}
Pelvic tuberculosis is a type of extrapulmonary tuberculosis. The disease is accompanied by clinical and laboratory findings which may be unspecific and present aspects of other diseases, including gynecological malignancies. In this report, the authors presented a case of pelvic tuberculosis associated with peritoneal tuberculosis in a young woman exhibiting imaging and tumor markers consistent with ovarian neoplasm. An intraoperative frozen section analysis detected atypical cells that were suggestive of ovarian borderline or malignant epithelial neoplasia. The pathological analysis showed granulomatous inflammation in the right ovary and fallopian tube with a pattern of mycobacteriosis that was consistent with the presence of mycobacteria morphologically compatible with Mycobacterium tuberculosis. The patient had a complete remission after the use of antituberculosis drugs.
\end{abstract}

\section{Introduction}

Ovarian cancer cases are often characterized by the presence of ascites, adnexal masses, and high CA-125 levels. Radical surgery is generally the primary treatment, and this can include removal of the uterus, ovaries, fallopian tubes, and pelvic lymph nodes [1]. It has been reported that pelvic tuberculosis can be misdiagnosed as ovarian cancer based on the clinical and laboratory similarities between these two diseases. Moreover, pelvic tuberculosis can also mimic other pathological conditions including bowel disease, cancer, and infectious diseases [2].

Pelvic tuberculosis is a type of extrapulmonary tuberculosis that is caused by Mycobacterium tuberculosis [3, 4]. The symptoms for pelvic tuberculosis are generally nonspecific and often include a low-grade fever, abdominal pain, infertility, and menstrual disorders. The latter disease is rare in the Western world, while it is more common in nonindustrialized countries $[5,6]$. However, due to greater opportunity for international travel and an increase in the incidence of immunosuppressive diseases such as AIDS, sporadic cases of peritoneal tuberculosis have been reported in greater number worldwide [1]. Risk factors include exposure to immigrants from countries with a high prevalence of the disease, urban poor, homeless, elderly individuals, and especially immunocompromised patients due to HIV infection $[5,7]$.

Here, we report a case of pelvic tuberculosis associated with peritoneal tuberculosis in a young woman exhibiting symptoms consistent with ovarian cancer.

\section{Case Presentation}

A 24-year-old woman presented to our Pelvic Mass Ambulatory Unit (Federal University of the Triângulo Mineiro (UFTM)) with a history of abdominal pain for the previous week that was associated with weight loss, fever, diarrhea, 


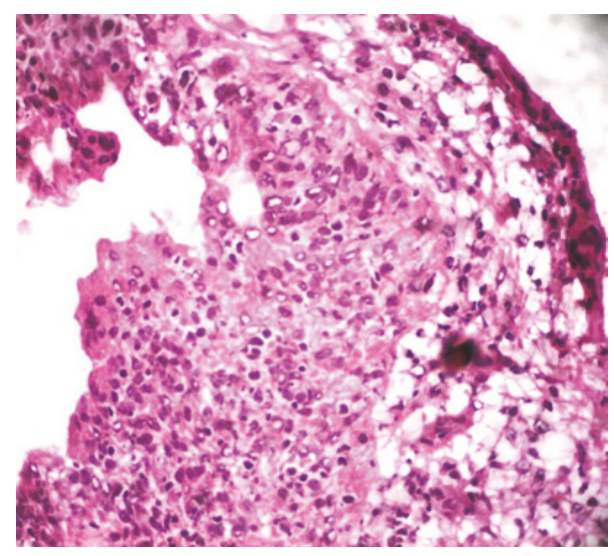

FIGURE 1: Intraoperative frozen section: atypical cells that were suggestive of ovarian borderline or malignant epithelial neoplasia.

nausea, and ascites. A chest X-ray was normal. Abdominal ultrasonography showed an enlarged right ovary $(3.2 \times 4.0$ $\times 4.0 \mathrm{~cm}$; volume, $27.6 \mathrm{ml}$ ), with heterogeneous echogenicity, cystic areas, and thick walls. Magnetic resonance imaging (MRI) showed a heterogeneous mass with well-defined edges involving the right ovary that measured approximately $5.0 \mathrm{~cm}$ in diameter and exhibited heterogeneous contrast enhancement. The left ovary and uterus were normal. Additional observations included enlarged iliac lymph nodes, smaller lesions in the posterior fornix, abdominal ascites, and diffuse enhancement of the peritoneum. CA-125 and CA-15.3 levels were also increased $(201 \mathrm{U} / \mathrm{mL}$ and $40.2 \mathrm{U} / \mathrm{ml}$, resp.).

An exploratory laparotomy was performed. The pelvis was frozen and the entire bowel and omentum were congested, similar to peritoneal carcinomatosis. During the procedure, biopsies were performed of the pelvic peritoneum, the fallopian tube, and the right ovary. An ascite sample was also collected. An intraoperative frozen section analysis detected atypical cells that were suggestive of ovarian borderline or malignant epithelial neoplasia (Figure 1). Despite these results, conservative surgery was performed based on the patient's desire to maintain her ability to become pregnant.

The pathological analysis showed granulomatous inflammation in the right ovary and fallopian tube with a pattern of mycobacteriosis that was consistent with the presence of mycobacteria morphologically compatible with Mycobacterium tuberculosis.

The patient was referred to the Infectious Diseases Service and received antituberculosis drugs. Six months later, the patient reported overall good health. Her CA-125 levels and an abdominopelvic ultrasound were both normal. Upon completion of the tuberculosis treatment, the patient was trying to become pregnant.

\section{Discussion}

Pelvic tuberculosis can present with nonspecific symptoms and similar laboratory and clinical findings to ovarian malignancy. Here, a patient with pelvic tuberculosis experienced pain and weight loss that were accompanied by increased tumor marker levels, the presence of ascites, and an ovarian mass. Surgery was necessary to confirm a diagnosis and treatment plan.

The presence of abdominopelvic pain, ascites, nausea, vomiting, and anorexia associated with imaging tests showing pelvic mass were reported in patients with abdominopelvic tuberculosis [4, 8-11] and may be associated with increased serum CA-125 levels [4, 8-10]. In these women, the association of these symptoms, imaging tests, and CA 125 levels mimicked ovarian cancer, delaying the tuberculosis diagnosis.

The treatment of pelvic tuberculosis is usually nonsurgical. It is difficult to diagnose pelvic tuberculosis prior to surgery, and currently there are no laboratory tests or imaging modalities that can differentiate this disease from advanced ovarian cancer [8]. Moreover, identification of a less invasive intervention for obtaining a differential diagnosis remains a major challenge. While a tuberculin skin test is an option, its low sensitivity may lead to false negatives in cases of abdominal tuberculosis $[4,12]$. The present patient had no previous history of tuberculosis, her family history was negative, and she denied any contact with diseased individuals. Serological testing for sexually transmitted diseases was also negative. For a patient with a history of tuberculosis, close contact with infected individuals, travel to, or residency in, endemic countries, and immunodeficiency state are key considerations [13].

It is important to note that pelvic and pulmonary tuberculosis are not always associated. A standard chest X-ray is often performed, and computed tomography of the abdomen and pelvis can show ascites and induration of the mesentery and omentum [14]. CA 125 levels have been found to be increased in only half of patients with early epithelial ovarian tumors and in two-thirds of patients with advanced tumors. Since CA 125 levels can also be increased with pelvic tuberculosis, these levels are not very useful for a differential diagnosis of pelvic tuberculosis versus ovarian neoplasms [1]. However, in areas where tuberculosis is endemic, the detection of high levels of CA-125 in premenopausal women should lead to further examination of a tuberculosis diagnosis [8]. In the present case, the levels of CA-125 and CA-15.3 were increased and the chest X-ray was normal.

A BAAR analysis of ascites also has a low sensitivity for a diagnosis of peritoneal tuberculosis. However, an advance in the analysis of ascitic fluid in this context has been the detection of adenosine deaminase (ADA). This enzyme is involved in the conversion of adenosine to inosine and is released by macrophages and lymphocytes during an immune response. In a previous study it was demonstrated that a cutoff level of $39 \mathrm{U} / \mathrm{I}$ for ADA levels provided high sensitivity and specificity for a diagnosis of abdominopelvic tuberculosis [15]. Further studies are needed to confirm the use and sensitivity of this assay.

Laparoscopy as a diagnostic tool in combination with biopsy and histology was found to be effective for establishing a diagnosis of pelvic tuberculosis. Laparoscopic biopsy is a sensitive and specific diagnostic procedure for abdominal peritonitis, and it is considered to be the gold standard method for clearly differentiating advanced ovarian cancer 
and peritoneal tuberculosis, especially in areas where tuberculosis is endemic [16]. However, the use of laparoscopic surgery has also been associated with higher complication rates in patients with pelvic tuberculosis, and laparoscopic findings can resemble those of metastatic ovarian cancer. Therefore, only a biopsy that shows caseating granulomas in a histopathological analysis can provide a definitive diagnosis of tuberculosis. Meanwhile, intraoperative frozen section analyses that reveal caseous necrosis with granulomatous lesions can potentially prevent unnecessary oophorectomies in young women $[17,18]$. In the present case, cellular atypia in the frozen sections indicated ovarian cancer.

Although frozen section has good accuracy for malignant and benign neoplasms, its efficiency in detecting borderline ovarian tumors is not high, perhaps because more extensive samples are needed $[19,20]$. Intraoperative frozen section sensitivity ranges from $86.1 \%$ to $92.5 \%, 98.2 \%$ to $100 \%$, and $44.8 \%$ to $87 \%$ in malignant, benign, and borderline tumors, respectively. Its specificity ranges from 98.5 to $100 \%, 87$ to $92.8 \%$, and 96.4 to $98.6 \%$, respectively [19-23]. Thus, conservative surgeries should be performed when intraoperative frozen section shows borderline tumor or malignant tumor in young patients wishing to preserve fertility.

The ability to provide a preoperative differential diagnosis of pelvic tuberculosis that mimics an ovarian tumor remains a challenge, especially in the absence of specific protocols, nonspecific symptoms, and the rarity of cases that characterize this disease. As demonstrated in the present case, conservative surgery should be performed when a diagnosis is uncertain, especially for patients who wish to become pregnant.

\section{Consent}

Consent was obtained from the patient for publication of this case report.

\section{Conflicts of Interest}

The authors declare no conflicts of interest.

\section{Acknowledgments}

The authors wish to acknowledge CNPq (Conselho Nacional de Desenvolvimento Científico e Tecnológico), FUNEPU (Fundação de Ensino e Pesquisa de Uberaba), and the FAPEMIG (Fundação de Amparo à Pesquisa do Estado de Minas Gerais).

\section{References}

[1] M. A. Gosein, D. Narinesingh, G. V. Narayansingh, N. A. Bhim, and P. A. Sylvester, "Peritoneal tuberculosis mimicking advanced ovarian carcinoma: an important differential diagnosis to consider," BMC Research Notes, vol. 6, article 88, 2013.

[2] C. Pina, M. Teixeira, D. Cruz, E. Ferreira, M. Mesquita, and A. Torgal, "Pelvic tuberculosis: the great simulator of gynaecologic malignancies," Arquivos de Medicina, vol. 22, no. 2-3, pp. 45-48, 2008 .
[3] M. I. Campagnolo, R. d. Reis, V. F. Oliveira, H. I. Mônego, and W. Rivoire, "Tuberculose Pélvica simulando tumor ovariano: a case report," Revista Brasileira de Ginecologia e Obstetrícia, vol. 22, no. 8, pp. 525-528, 2000.

[4] Q. Liu, Q. Zhang, Q. Guan, J.-F. Xu, and Q.-L. Shi, "Abdominopelvic tuberculosis mimicking advanced ovarian cancer and pelvic inflammatory disease: a series of 28 female cases," Archives of Gynecology and Obstetrics, vol. 289, no. 3, pp. 623629, 2014.

[5] W. P. Irvin Jr., L. W. Rice, and W. A. Andersen, "Abdominal tuberculosis mimicking metastatic ovarian carcinoma," Obstetrics and Gynecology, vol. 92, no. 4, part 2, article 709, 1998.

[6] T. A. Panoskaltsis, D. A. Moore, D. A. Haidopoulos, and A. G. McIndoe, "Tuberculous peritonitis: part of the differential diagnosis in ovarian cancer," American Journal of Obstetrics \& Gynecology, vol. 182, no. 3, pp. 740-742, 2000.

[7] A. Mahdavi, V. K. Malviya, and B. R. Herschman, "Peritoneal tuberculosis disguised as ovarian cancer: an emerging clinical challenge," Gynecologic Oncology, vol. 84, no. 1, pp. 167-170, 2002.

[8] F. Yassaee and F. Farzaneh, "Familial tuberculosis mimicking advanced ovarian cancer," Infectious Diseases in Obstetrics and Gynecology, vol. 2009, Article ID 736018, 5 pages, 2009.

[9] C.-H. Wu, C.-C. ChangChien, C.-W. Tseng, H.-Y. Chang, Y.C. $\mathrm{Ou}$, and H. Lin, "Disseminated peritoneal tuberculosis simulating advanced ovarian cancer: a retrospective study of 17 cases," Taiwanese Journal of Obstetrics and Gynecology, vol. 50, no. 3, pp. 292-296, 2011.

[10] S. Imtiaz, N. Siddiqui, M. Ahmad, and A. Jahan, "Pelvicperitoneal tuberculosis mimicking ovarian cancer," Journal of the College of Physicians and Surgeons Pakistan, vol. 22, no. 2, pp. 113-115, 2012.

[11] E. Yebouet, M. M. Olivier, S. Koui, and S. R. Bankole, "Ovarian tuberculosis mimicking a malignant tumour," African Journal of Paediatric Surgery, vol. 12, no. 2, pp. 155-157, 2015.

[12] N. H. Gilinsky, I. N. Marks, R. E. Kottler, and S. K. Price, "Abdominal tuberculosis. A 10-year review," South African Medical Journal, vol. 64, no. 22, pp. 849-857, 1983.

[13] R. Lermann, A. Borges, and G. Pinto, "Genital tuberculosis-a case report," Revista Iberoamericana de Fertilidad y Reproduccion Humana, vol. 28, no. 4, article 6, 2011.

[14] C. Clancy, Y. Bokhari, P. M. Neary, and M. Joyce, "Diagnosing peritoneal tuberculosis," BMJ Case Reports, vol. 2013, 2013.

[15] A. Riquelme, M. Calvo, F. Salech et al., "Value of adenosine deaminase (ADA) in ascitic fluid for the diagnosis of tuberculous peritonitis: a meta-analysis," Journal of Clinical Gastroenterology, vol. 40, no. 8, pp. 705-710, 2006.

[16] A. Tinelli, A. Malvasi, D. Vergara et al., "Abdominopelvic tuberculosis in gynaecology: laparoscopical and new laboratory findings," Australian and New Zealand Journal of Obstetrics and Gynaecology, vol. 48, no. 1, pp. 90-95, 2008.

[17] T. Oge, S. S. Ozalp, O. T. Yalcin et al., "Peritoneal tuberculosis mimicking ovarian cancer," European Journal of Obstetrics Gynecology and Reproductive Biology, vol. 162, no. 1, pp. 105-108, 2012.

[18] T. A. Panoskaltsis, D. A. Moore, D. A. Haidopoulos, and A. G. McIndoe, "Tuberculous peritonitis: part of the differential diagnosis in ovarian cancer," American Journal of Obstetrics and Gynecology, vol. 182, no. 3, pp. 740-742, 2000.

[19] P. G. Rose, R. B. Rubin, B. E. Nelson, R. E. Hunter, and F. R. Reale, "Accuracy of frozen-section (intraoperative consultation) 
diagnosis of ovarian tumors," American Journal of Obstetrics and Gynecology, vol. 171, no. 3, pp. 823-826, 1994.

[20] E. L. K. Yeo, K. M. Yu, N. C. Poddar, P. K. Hui, and L. C. H. Tang, "The accuracy of intraoperative frozen section in the diagnosis of ovarian tumors," Journal of Obstetrics and Gynaecology Research, vol. 24, no. 3, pp. 189-195, 1998.

[21] F. C. M. Twaalfhoven, A. A. W. Peters, J. B. Trimbos, J. Hermans, and G. J. Fleuren, "The accuracy of frozen section diagnosis of ovarian tumors," Gynecologic Oncology, vol. 41, no. 3, pp. 189192, 1991.

[22] S. Ilvan, R. Ramazanoglu, E. Ulker Akyildiz, Z. Calay, T. Bese, and N. Oruc, "The accuracy of frozen section (intraoperative consultation) in the diagnosis of ovarian masses," Gynecologic Oncology, vol. 97, no. 2, pp. 395-399, 2005.

[23] V. Wootipoom, C. Dechsukhum, J. Hanprasertpong, and A. Lim, "Accuracy of intraoperative frozen section in diagnosis of ovarian tumors," Journal of the Medical Association of Thailand, vol. 89, no. 5, pp. 577-582, 2006. 


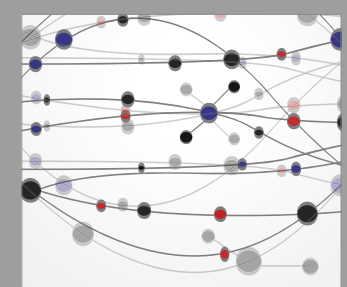

The Scientific World Journal
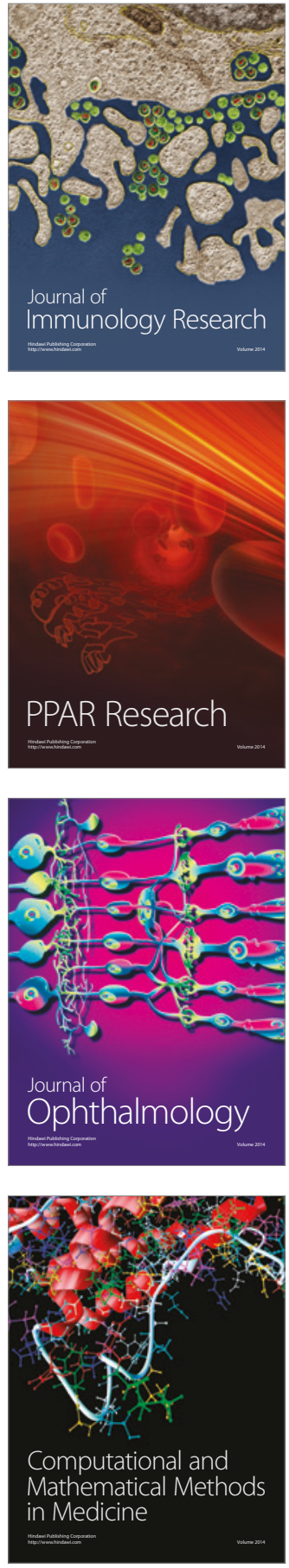

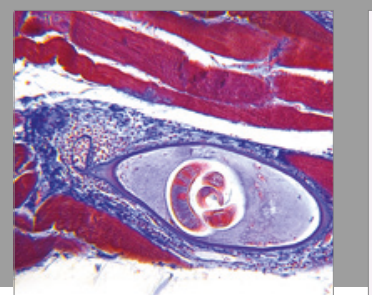

Gastroenterology Research and Practice
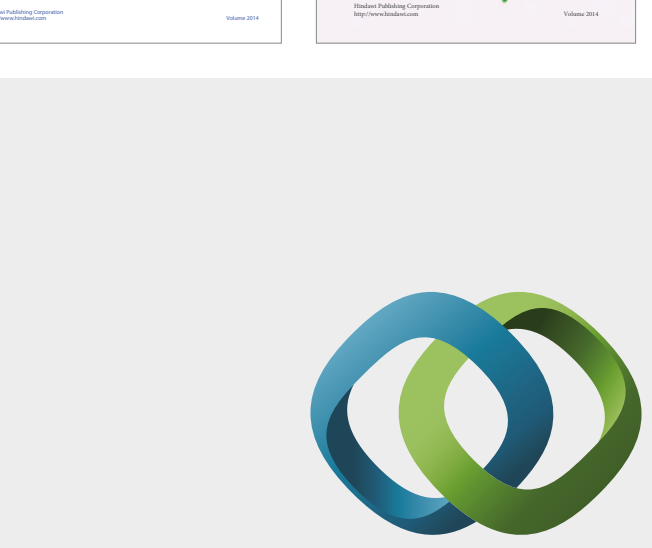

\section{Hindawi}

Submit your manuscripts at

https://www.hindawi.com
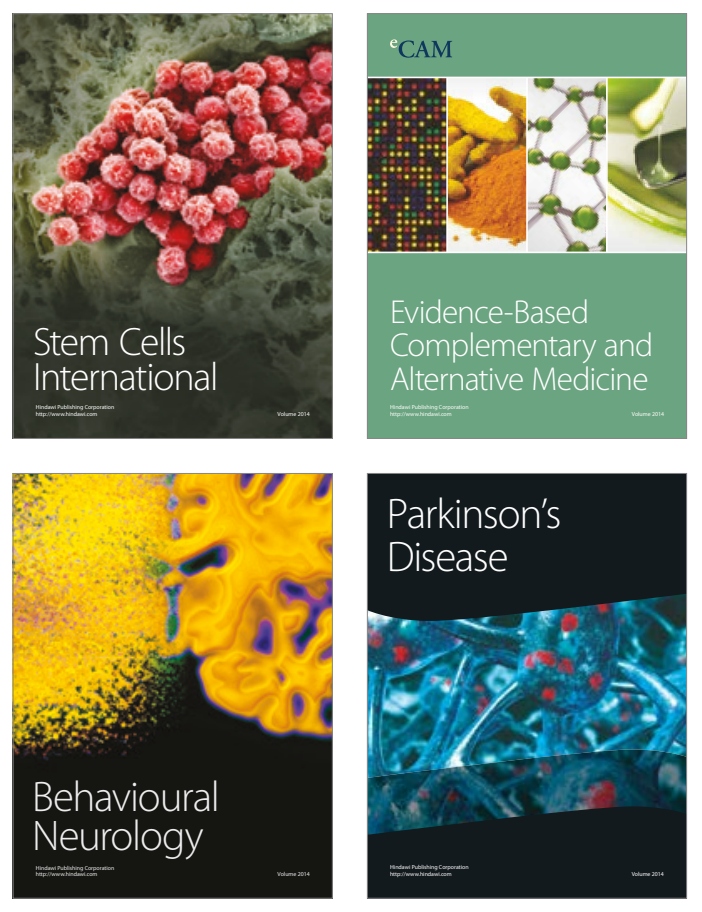
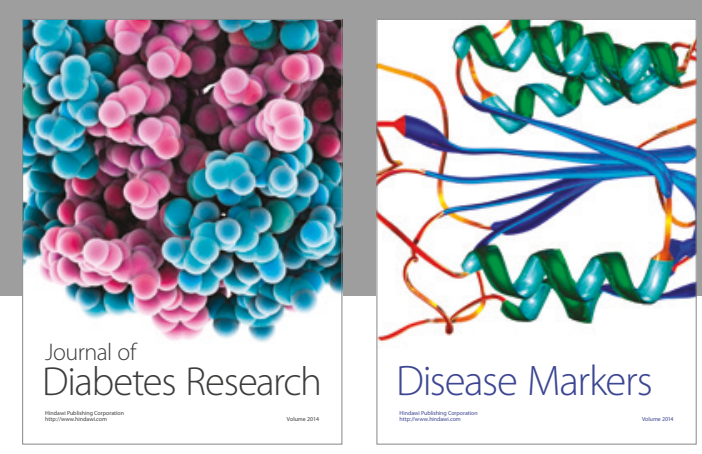

Disease Markers
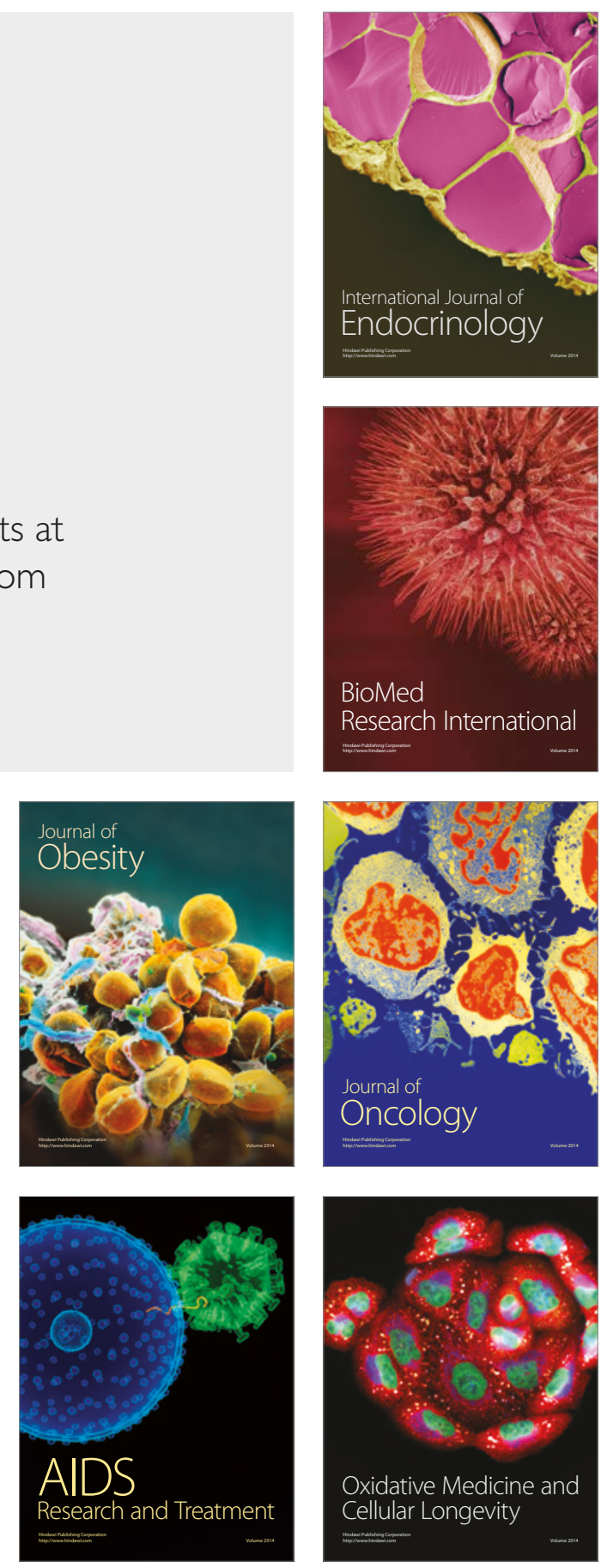